\title{
Coefficients and Roots of the Polynomials which Define the Derivatives of the Exponential of $(-\epsilon / T)$
}

\author{
By Edwin S. Campbell, E. M. Fishbach, \\ and $\mathrm{J}$. O. Hirschfelder
}

The $n$-th derivative of $\exp (-\epsilon / T)$ can be shown to be of the form

$$
\begin{aligned}
d^{n} \exp [-\epsilon / T] / d T^{n} & =T^{-n} \exp [-\epsilon / T] W_{n}[\epsilon / T] \\
W_{n}[\epsilon / T] & =\sum_{l=1}^{n} b_{n, l}[\epsilon / T]^{l} .
\end{aligned}
$$

The $b_{n, l}$ are constant coefficients in a homogeneous polynomial of the $n$-th order in $[\epsilon / T]$. These polynomials attain added importance from their relation to the Laguerre polynomials [1]

$$
W_{n}[\epsilon / T]=(-1)^{n}\left\{L_{n}[\epsilon / T]-n L_{n-1}[\epsilon / T]\right\}
$$

$L_{n}[\epsilon / T]$ : the $n$-th order Laguerre polynomial.

The zeros of these polynomials are of interest since they locate the extreme values of the derivatives of exp $(-\epsilon / T)$. Furthermore, an accurate, simple computation of the $W_{n}$ for values of $(\epsilon / T)$. which occur in physical problems sometimes requires use of the polynomial roots. This occurs whenever the direct evaluation of $W_{n}[\epsilon / T]$ by synthetic division introduces at intermediate steps of the calculation numbers which are larger than the value of the polynomial. In such cases the subtraction of these larger numbers can require the use of considerably more than the usual guard figures which allow for the effect of ordinary round-off error. Fortunately the occurrence of this disastrous subtraction in synthetic division does not imply the occurrence when the polynomial is calculated using its roots

$$
W_{n}[\epsilon / T]=\prod_{l=1}^{n}\left[\epsilon / T-r_{n, l}\right]
$$

$r_{n, l}:$ the $l$-th root of $W_{n}$.

The polynomial coefficients, $b_{n, l}$, are integers. Table I lists all of their non-zero digits which were computed from the recursion relations

$$
\begin{aligned}
& b_{n, 1}=(-1)^{n-1} n ! \\
& b_{n, l}=-(n+l-1) b_{n-1, l}+b_{n-1, l-1}(2 \leq l \leq n-1) \\
& b_{n, n}=1 .
\end{aligned}
$$

The values in the Table were checked numerically by the distinct relations

$$
(n-l) b_{n, l}=-n(n-1) b_{n-1, l} \text {. }
$$

Received 16 September 1957. 
TABLE I. Exact Values of the Coefficients, $b_{n, l}$

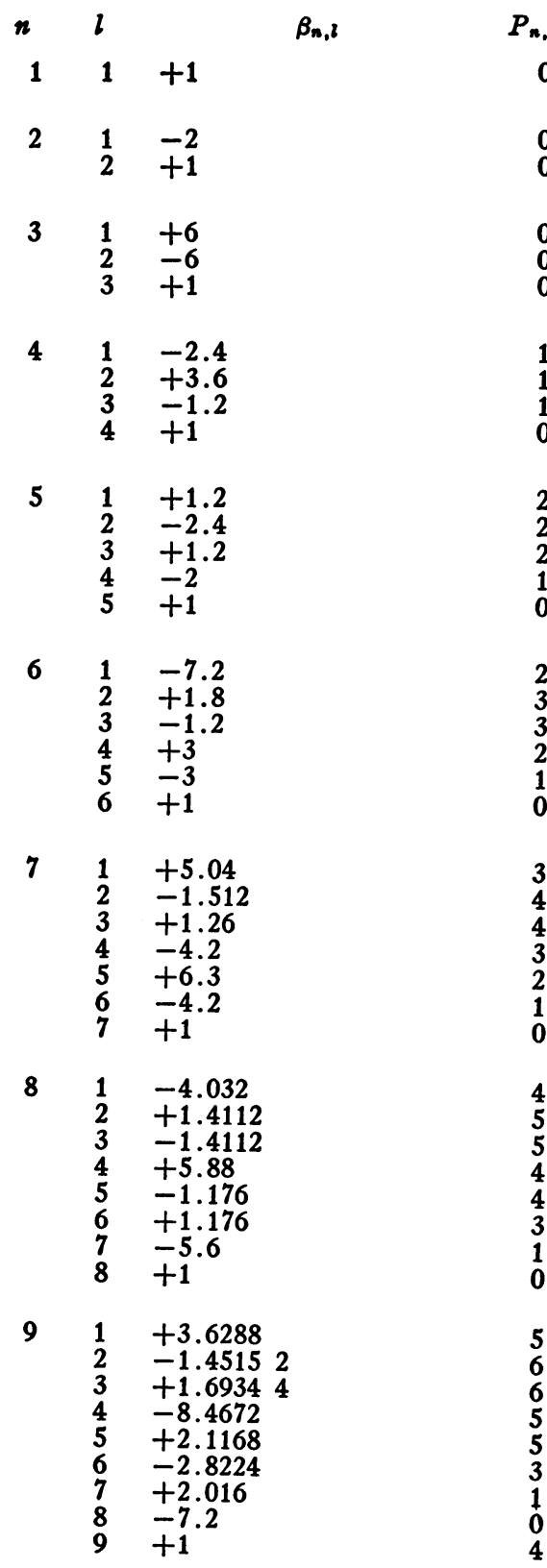

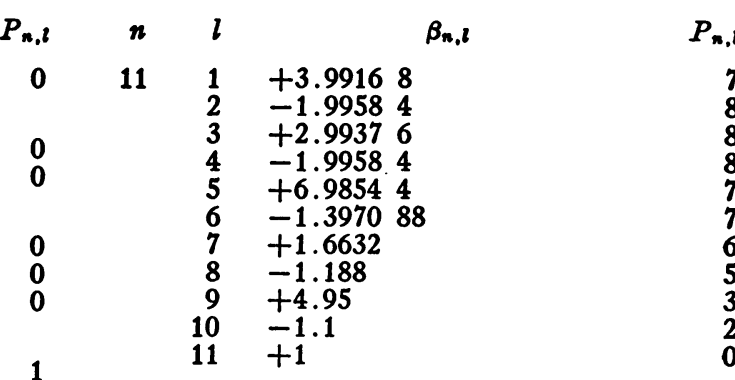

$P_{n, l}$

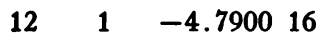

$+2.6345088$

$-4.390848$

$+3.293136$

$-1.3172544$

$+3.0735936$

$-4.390848$

$+3.9204$

$-2.178$

$10+7.26$

$11 \quad-1.32$

$11+1$

13

$\begin{array}{lll}1 & +6.2270208 \\ 2 & -3.7362 & 1248\end{array}$

$+6.84972288$

$-5.7081024$

$+2.56864608$

$-6.84972288$

$+1.14162048$

$-1.2231648$

$+8.4942$

$10-3.7752$

$11+1.0296$

$12-1.56$

$\begin{array}{llll}14 & 1 & -8.7178 & 2912\end{array}$

-1.56
+1
-8.71782912
+5.666588928

$-1.1333177856$

$+1.0388746368$

$-5.194373184$

$+1.5583119552$

$-2.968213248$

$+3.71026656$

$-3.0918888$

$+1.717716$

$-6.24624$

$+1.4196$

$-1.82$

$14+1$

$+1.307674368$

$-9.153720576$

$+1.9833061248$

$-1.9833061248$

$+1.09081836864$

$-3.6360612288$

$+7.791559776$

$-1.113079968$

$+1.08216108$

$-7.2144072$

$+3.279276$

$-9.9372$

$+1.911$

$-2.1$

$15+1$

$8+3.2$

$10+1$ 
TABLE I. Exact Values of the Coefficients, $b_{n, l}$-Continued

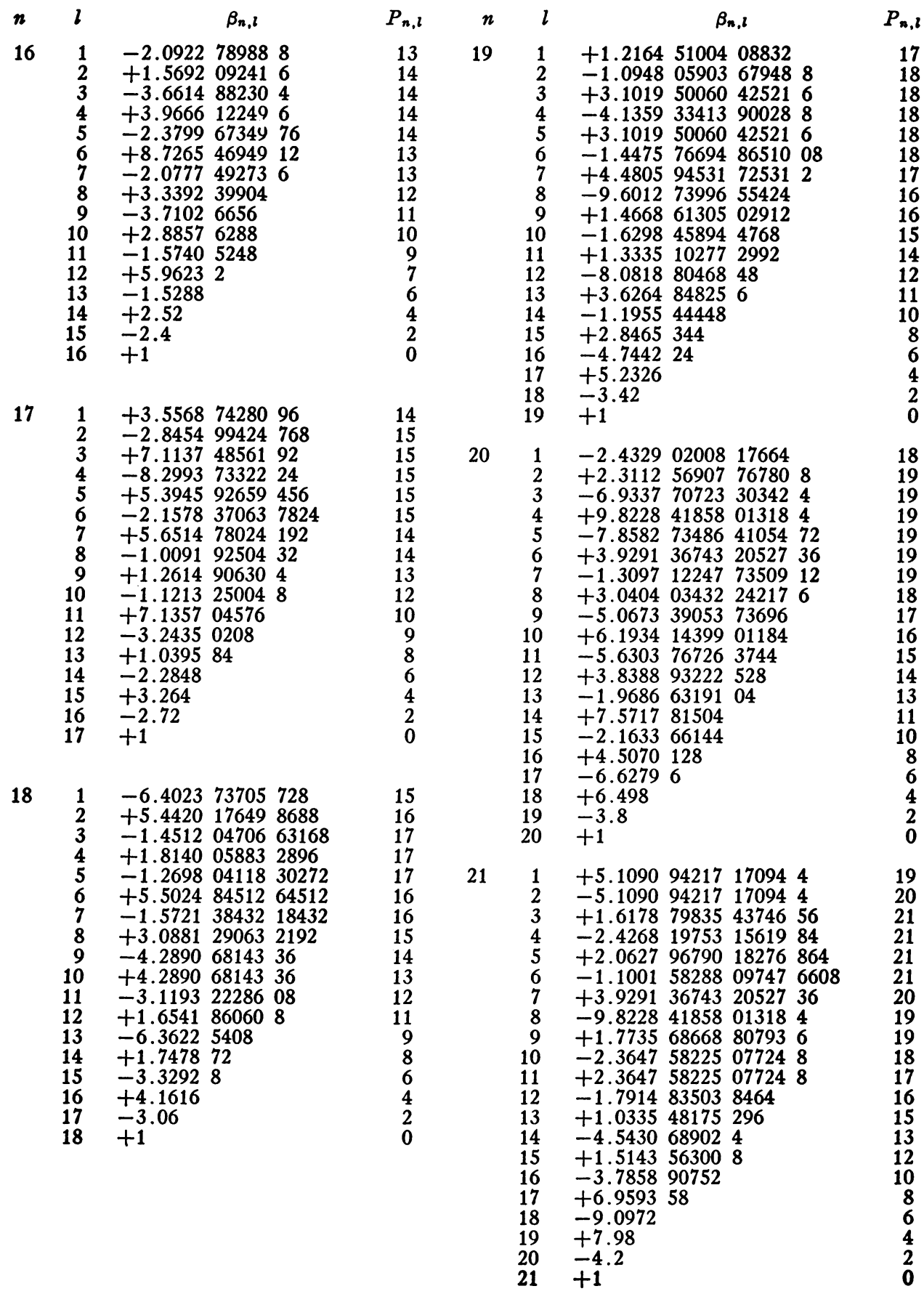


TABLE I. Exact Values of the Coefficients, $b_{n, t}$-Continued

$\boldsymbol{n}$

22

23
1
2
3
4
5
6
7
8
9
10
11
12
13
14
15
16
17
18
19
20
21
22

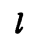

1

1
2
3
4
5
6
7
8
9
10
11
12
13
14
15
16
17
18
19
20
21
22
23

1

4

4

6

7

8

9

11

12

13

14

15

16

17

18

20 $\beta_{n, l}$

$P_{n, l}$

$\begin{array}{llll}-1.1240 & 00727 & 77760768\end{array}$

$+1.180200764166488064$

$-3.93400254722162688$

$+6.22883736643424256$

-5.605953629790818304

$+3.1767070568814637056$

$-1.2101741169072242688$

$+3.24153781314435072$

$-6.3029901922251264$

$+9.1043191665474048$

$-9.9319845453244416$

$+8.276653787770368$

$-5.3055472998528$

$+2.623622291136$

$-9.99475158528$

$+2.91513587904$

$-6.430446792$

$+1.0507266$

$-1.22892$

$+9.702$

$-4.62$

$+1$

$+2.585201673888497664$

$-2.8437218412773474304$

+9.953026444 4707160064

$-1.6588377407451193344$

$+1.57589585370786336768$

$-9.455375122 \quad 24718020608$

$+3.82717564471909675008$

$-1.09347875563402764288$

$+2.278080740904224256$

$-3.543681152517682176$

$+4.187986816611806208$

$-3.80726074237436928$

$+2.6846069337255168$

$-1.47505875479424$

$+6.3216803776896$

$-2.1072 \quad 267925632$

$+5.42301012792$

$-1.0633353192$

$+1.5545838$

$-1.636404$

$+1.16886$

$-5.06$

$+1$

$-6.2044840173323943936$

$+7.13515661993225355264$

$-2.616224093975159635968$

$+4.578392164456529362944$

$-4.578392164456529362944$

$+2.8996483708224685965312$

$-1.2427064446382008270848$

$+3.772501706937395367936$

$-8.38333712652754526208$

$+1.39722285442125754368$

$-1.77828363289978232832$

$+1.7513399414922098688$

$-1.347184570378622976$

$+8.1423243264642048$

$-3.877297298316288$

$+1.453986486868608$

$-4.2764308437312$

$+9.78268493664$

$-1.7162605152$

$+2.25823752$
21

22

22

22

22

22

22

21

20

19

18

17

16

15

13

12

10

9

7

2

22

23

23

24

24

23

23

22

21

20

19

18

17

15

14

12

11

7

5

2

23

24

25

25

25

25

25

24

23

23

22

21

20

18

17

16

14

12

11 
TABLE I. Exact Values of the Coefficients, $b_{n, l}$-Continued

$n$

24

$\begin{array}{ll}21 & -2.1507024 \\ 22 & +1.39656 \\ 23 & -5.52 \\ 24 & +1\end{array}$

$\beta_{n, l}$

$P_{n, l}$

7

5

25

$$
\begin{array}{lllll}
+1.5511 & 21004 & 33309 & 85984 & \\
-1.8613 & 45205 & 19971 & 83180 & 8 \\
+7.1351 & 56619 & 93225 & 35526 & 4 \\
-1.3081 & 12046 & 98757 & 98179 & 84 \\
+1.3735 & 17649 & 33695 & 88088 & 832 \\
-9.1567 & 84328 & 91305 & 87258 & 88 \\
+4.1423 & 54815 & 46066 & 94236 & 16 \\
-1.3314 & 71190 & 68378 & 66004 & 48 \\
+3.1437 & 51422 & 44782 & 94732 & 8 \\
-5.5888 & 91417 & 68503 & 01747 & 2 \\
+7.6212 & 15569 & 57049 & 56928 \\
+8.0831 & 07422 & 27173 & 7856 \\
+6.7359 & 22851 & 89311 & 488 \\
+4.4412 & 67814 & 43502 & 08 \\
+2.3263 & 78378 & 98977 & 28 \\
+9.6932 & 43245 & 79072 & \\
+3.2073 & 23132 & 7984 & \\
+8.3851 & 58517 & 12 & & \\
+1.7162 & 60515 & 2 & &
\end{array}
$$

26

$-4.03291461126605635584$

$+5.0411432640825704448$

$-2.01645730563302817792$

$+3.86487650246330400768$

$-4.251364152709634408448$

$+2.9759549068967440859136$

$-1.417121384236544802816$

$+4.80809041080256272384$

$-1.20202260270064068096$

$+2.27048713843454350848$

$-3.30252674681388146688$

$+3.752871303197592576$

$-3.36796142594655744$

$+2.4056867328189696$

$-1.3746781330394112$

$+6.300608109763968$

$-2.3164000403544$

$+6.81294129516$

$-1.5936704784$

$+2.935708776$

$-4.19386968$

$+4.53882$

$-3.588$

$+1.95$

$-6.5$

$+1$

$+1.0888869450418352160768$

-1.4155 5302855438578089984

$+5.898137618976607420416$

$-1.1796275237953214840832$

$+1.35657165236461970669568$

-9.9481 9211734054451576832

$+4.97409605867027225788416$

$-1.7764628780965258063872$

$+4.687888150532498655744$
25

26

26

27

27

26

26

25

24

23

22

21

20

19

17

16

14

13

11

7

5

2
0

26

27

28

28

28

28

28

27

26

25

24

23

22

21

19

18

16

15

13

11

9

5

2

28

29

29

30

30

29

29

29 
TABLE I. Exact Values of the Coefficients, $b_{n, l}$-Continued

$-5.3140942102248 \quad 18$

$\begin{array}{ll}+1.398445844796 & 17\end{array}$

$\begin{array}{lll}-2.94409651536 & 15\end{array}$

$+4.9068275256 \quad 13$

$-6.37250328$

$+6.29694$

$-4.563$

$+2.2815$

$-7.02$

$+1$

-3.0488 83446 11713860501504

$+4.115992652258137116770304$

$-1.7835968159785260839337984$

$+3.71582669995526267486208$

$-4.458992039946315209834496$

$+3.4185605639588416608731136$

$-1.7906745811212980128382976$

+6.715029679204867548143616

$-1.86528602200135209670656$

+3.93782604644729887082496

$-6.44371534873194360680448$

$+8.2987243127608364633088$

$-8.511512115652139962368$

$+7.0149825129001153536$

$-4.6766550086000769024$

$+2.5331881296583749888$

$-1.117582998378694848$

$+4.0174552229299488$

$-1.17469450962864$

$+2.7821712070152$

$-5.299373727648$

$+8.0293541328$

$-9.52097328$

$+8.62407$

$-5.74938$

$+2.65356$

$-7.56$

$+1$

\section{$+8.841761993739701954543616$}

-1.2378 4667912355827363610624

$+5.57031005605601223136247808$

$-1.206900512145469316795203584$

$+1.50862564018183664599400448$

$-1.206900512145469316795203584$

+6.60921709032042721102135296

$-2.59647814262588211861553152$

$+7.5730612493254895126286336$

$-1.6829024998501087805841408$

$+2.9068315906501878937362432$

$-3.963861259977528946003968$

+4.319592398 69346103090176

$-3.7974438669832624447488$

$+2.712459904988044603392$

$-1.582268277909692685312$

$+7.5623116223625018048$

$-2.965612400926471296$

$+9.5385194181845568$

29

30

31

31

31

31

30

30

29

28

26

25

24

23

22

20

19
17

15

13

11

9

5 
Table I. Exact Values of the Coefficients, $b_{n, t}$-Continued

\begin{tabular}{|c|c|c|c|c|}
\hline$n$ & $l$ & & $\beta_{n, l}$ & $P_{n, l}$ \\
\hline 29 & $\begin{array}{l}20 \\
21 \\
22 \\
23 \\
24 \\
25 \\
26 \\
27 \\
28 \\
29\end{array}$ & $\begin{array}{ll}-2.5101 & 36688 \\
+5.3788 & 64333 \\
-9.3140 & 50794 \\
+1.2885 & 05050 \\
+1.4005 & 48968 \\
+1.1671 & 2414 \\
+1.1823 & 024 \\
-7.10636 \\
+3.0693 \\
-8.12 \\
+1\end{array}$ & $\begin{array}{l}995936 \\
56272 \\
048 \\
56\end{array}$ & $\begin{array}{r}19 \\
17 \\
15 \\
14 \\
12 \\
10 \\
7 \\
5 \\
2 \\
0\end{array}$ \\
\hline 30 & $\begin{array}{l}1 \\
2 \\
3 \\
4 \\
5 \\
6 \\
7 \\
8 \\
9 \\
10 \\
11 \\
12 \\
13 \\
14 \\
15 \\
16 \\
17 \\
18 \\
19 \\
20 \\
21 \\
22 \\
23 \\
24 \\
25 \\
26 \\
27 \\
28 \\
29 \\
30\end{array}$ & $\begin{array}{ll}-2.6525 & 28598 \\
+3.8461 & 66467 \\
-1.7948 & 77684 \\
+4.0384 & 74790 \\
-5.2500 & 17227 \\
+4.3750 & 14356 \\
-2.5000 & 08203 \\
+1.0267 & 89083 \\
-3.1374 & 11089 \\
+7.3206 & 25874 \\
-1.3310 & 22886 \\
+1.9158 & 66275 \\
-2.2106 & 14933 \\
+2.0648 & 60102 \\
-1.5732 & 26744 \\
+9.8326 & 67155 \\
-5.0609 & 31624 \\
+2.1500 & 68990 \\
-7.5441 & 01721 \\
+2.1838 & 18919 \\
+5.1995 & 68855 \\
+1.0129 & 03023 \\
-1.6014 & 27705 \\
+2.0307 & 96003 \\
+2.0307 & 96003 \\
+1.5621 & 50772 \\
+8.9011 & 44 \\
+3.5322 & \\
+8.7 & \\
+1 & \end{array}$ & $\begin{array}{llll}12191 & 05863 & 63084 & 8 \\
27677 & 03502 & 26472 & 96 \\
72915 & 94967 & 72354 & 048 \\
64060 & 88677 & 37796 & 608 \\
83279 & 15280 & 59135 & 5904 \\
52732 & 62733 & 82612 & 992 \\
72990 & 07276 & 47207 & 424 \\
67478 & 06559 & 97960 & 192 \\
00627 & 42266 & 60433 & 92 \\
34797 & 31955 & 41012 & 48 \\
24508 & 60355 & 52911 & 36 \\
65580 & 56572 & 35251 & 2 \\
44900 & 65275 & 79136 & \\
67214 & 89543 & 3216 & \\
89306 & 58699 & 6736 & \\
58166 & 16872 & 96 & \\
19644 & 35155 & 2 & \\
67169 & 16896 & & \\
65505 & 856 & & \\
42646 & 432 & & \\
77729 & 6 & & \\
85272 & & & \end{array}$ & $\begin{array}{l}32 \\
33 \\
34 \\
34 \\
34 \\
34 \\
34 \\
34 \\
33 \\
32 \\
32 \\
31 \\
30 \\
29 \\
28 \\
26 \\
25 \\
24 \\
22 \\
21 \\
19 \\
18 \\
16 \\
14 \\
12 \\
10 \\
7 \\
5 \\
2 \\
0\end{array}$ \\
\hline
\end{tabular}

Legend: $W_{n}(x)=\sum_{l=1}^{n} b_{n, l} x^{l}$

$$
b_{n, l}=\beta_{n, l} \times 10^{P_{n, l},} \quad 1 \leqq \beta_{n, l}<10
$$

TABLE II. Roots of $W_{n}(\epsilon / T)$

\begin{tabular}{|c|c|c|c|c|c|}
\hline$n$ & $l$ & $r_{n, l}$ & & $C$ & $e_{n, 2}$ \\
\hline 2 & 1 & 2.0000000000 & 0000 & 0 & 0 \\
\hline $\begin{array}{l}3 \\
3\end{array}$ & $\begin{array}{l}1 \\
2\end{array}$ & 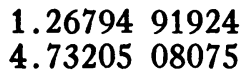 & $\begin{array}{l}3112 \\
6888\end{array}$ & $\begin{array}{l}0 \\
0\end{array}$ & \\
\hline $\begin{array}{l}4 \\
4 \\
4\end{array}$ & $\begin{array}{l}1 \\
2 \\
3\end{array}$ & $\begin{array}{ll}0.93582 & 22275 \\
3.30540 & 72893 \\
7.75877 & 04831\end{array}$ & $\begin{array}{l}2409 \\
3227 \\
4363\end{array}$ & $\begin{array}{l}0 \\
0 \\
0\end{array}$ & \\
\hline
\end{tabular}


TABLE II. Roots of $W_{n}(\epsilon / T)$-Continued

\begin{tabular}{|c|c|c|c|c|}
\hline$n$ & $l$ & $r_{n, l}$ & $C$ & $e_{n}$ \\
\hline $\begin{array}{l}5 \\
5 \\
5 \\
5\end{array}$ & $\begin{array}{l}1 \\
2 \\
3 \\
4\end{array}$ & $\begin{array}{rrr}0.74329 & 19279 & 8143 \\
2.57163 & 50076 & 4627 \\
5.73117 & 87516 & 8905 \\
10.95389 & 43126 & 8321\end{array}$ & $\begin{array}{l}0 \\
0 \\
0 \\
1\end{array}$ & $\begin{array}{r}5 \\
5 \\
50 \\
6\end{array}$ \\
\hline $\begin{array}{l}6 \\
6 \\
6 \\
6 \\
6\end{array}$ & $\begin{array}{l}1 \\
2 \\
3 \\
4 \\
5\end{array}$ & $\begin{array}{rrr}0.61703 & 08532 & 7825 \\
2.11296 & 59585 & 7838 \\
4.61083 & 31510 & 1760 \\
8.39906 & 69712 & 0486 \\
14.26010 & 30659 & 2081\end{array}$ & $\begin{array}{l}0 \\
0 \\
0 \\
0 \\
1\end{array}$ & $\begin{array}{l}50 \\
50 \\
50 \\
50 \\
10\end{array}$ \\
\hline $\begin{array}{l}7 \\
7 \\
7 \\
7 \\
7 \\
7\end{array}$ & $\begin{array}{l}1 \\
2 \\
3 \\
4 \\
5 \\
6\end{array}$ & $\begin{array}{rrr}0.52766 & 81217 & 1117 \\
1.79629 & 98096 & 4345 \\
3.87664 & 15204 & 7699 \\
6.91881 & 65667 & 0471 \\
11.23461 & 04290 & 8311 \\
17.64596 & 35523 & 8068\end{array}$ & $\begin{array}{l}0 \\
0 \\
0 \\
0 \\
1 \\
1\end{array}$ & $\begin{array}{r}50 \\
50 \\
50 \\
50 \\
6 \\
16\end{array}$ \\
\hline $\begin{array}{l}8 \\
8 \\
8 \\
8 \\
8 \\
8 \\
8\end{array}$ & $\begin{array}{l}1 \\
2 \\
3 \\
4 \\
5 \\
6 \\
7\end{array}$ & $\begin{array}{rrr}0.46102 & 42198 & 0496 \\
1.56358 & 61896 & 5431 \\
3.35205 & 05025 & 3674 \\
5.91629 & 72490 & 2042 \\
9.42069 & 93830 & 2156 \\
14.19416 & 55480 & 0748 \\
21.09217 & 69079 & 5447\end{array}$ & $\begin{array}{l}0 \\
0 \\
0 \\
0 \\
0 \\
1 \\
1\end{array}$ & $\begin{array}{l}50 \\
50 \\
50 \\
50 \\
50 \\
10 \\
22\end{array}$ \\
\hline $\begin{array}{l}9 \\
9 \\
9 \\
9 \\
9 \\
9 \\
9 \\
9\end{array}$ & $\begin{array}{l}1 \\
2 \\
3 \\
4 \\
5 \\
6 \\
7 \\
8\end{array}$ & $\begin{array}{rrr}0.40938 & 35732 & 0319 \\
1.38496 & 31848 & 0312 \\
2.95625 & 45561 & 6887 \\
5.18194 & 31010 & 4007 \\
8.16170 & 96881 & 4582 \\
12.07005 & 51268 & 3715 \\
17.24973 & 55261 & 4898 \\
24.58595 & 52436 & 5281\end{array}$ & $\begin{array}{l}0 \\
0 \\
0 \\
0 \\
0 \\
1 \\
1 \\
1\end{array}$ & $\begin{array}{r}5 \\
5 \\
5 \\
5 \\
5 \\
7 \\
15 \\
30\end{array}$ \\
\hline $\begin{array}{l}10 \\
10 \\
10 \\
10 \\
10 \\
10 \\
10 \\
10 \\
10\end{array}$ & $\begin{array}{l}1 \\
2 \\
3 \\
4 \\
5 \\
6 \\
7 \\
8 \\
9\end{array}$ & $\begin{array}{rrr}0.36817 & 84529 & 4174 \\
1.24335 & 79621 & 4047 \\
2.64603 & 38413 & 8420 \\
4.61688 & 25146 & 3485 \\
7.22178 & 65393 & 9663 \\
10.56732 & 08077 & 4184 \\
14.83591 & 45152 & 6107 \\
20.38218 & 19854 & 4899 \\
28.11834 & 33810 & 4993\end{array}$ & $\begin{array}{l}0 \\
0 \\
0 \\
0 \\
0 \\
1 \\
1 \\
1 \\
1\end{array}$ & $\begin{array}{r}5 \\
5 \\
5 \\
50 \\
50 \\
56 \\
110 \\
207 \\
39\end{array}$ \\
\hline $\begin{array}{l}11 \\
11 \\
11 \\
11 \\
11 \\
11 \\
11 \\
11 \\
11 \\
11\end{array}$ & $\begin{array}{r}1 \\
2 \\
3 \\
4 \\
5 \\
6 \\
7 \\
8 \\
9 \\
10\end{array}$ & $\begin{array}{rrr}0.33452 & 86763 & 2476 \\
1.12825 & 33558 & 7666 \\
2.39586 & 99247 & 4731 \\
4.16684 & 09879 & 2878 \\
6.48735 & 30313 & 8081 \\
9.42835 & 48133 & 3561 \\
13.10172 & 35803 & 6780 \\
17.69648 & 75668 & 4621 \\
23.57778 & 70883 & 6019 \\
31.68280 & 09748 & 3192\end{array}$ & $\begin{array}{l}0 \\
0 \\
0 \\
0 \\
0 \\
0 \\
1 \\
1 \\
1 \\
1\end{array}$ & $\begin{array}{r}5 \\
5 \\
5 \\
5 \\
5 \\
5 \\
9 \\
16 \\
28 \\
50\end{array}$ \\
\hline
\end{tabular}


TABLE II. Roots of $W_{n}(\epsilon / T)$-Continued

$\begin{array}{lr}n & \\ 12 & \\ 12 & \\ 12 & \\ 12 & \\ 12 & 5 \\ 12 & \\ 12 & 6 \\ 12 & 8 \\ 12 & 9 \\ 12 & 10 \\ 12 & 11\end{array}$

$\begin{array}{rll}r_{n, l} & \\ 0.30652 & 67021 & 3005 \\ 1.03279 & 73987 & 7972 \\ 2.18961 & 19419 & 6840 \\ 3.79904 & 76060 & 5497 \\ 5.89491 & 11715 & 0275 \\ 8.52729 & 20080 & 7027 \\ 11.77100 & 65654 & 9068 \\ 15.74226 & 02870 & 2615 \\ 20.63580 & 56686 & 4129 \\ 26.82634 & 99493 & 7082 \\ 35.27439 & 07009 & 6510\end{array}$

C

0

0

0

0

0

0

1

1

1

1

$e_{n, 6}$
5
5
5
5
5
5
7
12
213
36
62

$\begin{array}{lllll}13 & 1 & 0.28285 & 83482 & 3992\end{array}$

0.95232604136462

2.01649213857771

3.49235406977799

5.40549102001572

$\begin{array}{llll}7.79281 & 39404 & 1242\end{array}$

10.70738868898909

14.22715236378996

$18.4719966342 \quad 2982$

$23.64178 \quad 37524 \quad 0122$

30.12005862610650

38.88928437609550

0

5

$0 \quad 5$

$0 \quad 50$

$0 \quad 50$

$0 \quad 5$

$0 \quad 5$

16

1

10

171

279

455

76

$\begin{array}{lllll}14 & 1 & 0.26258839817108\end{array}$

0.88355030738774

1.86903381519979

3.23241869945120

4.99357560707419

7.18061049410079

9.83280825102000

13.00562240025865

16.77961364117585

$21.27791 \quad 17554 \quad 4831$

$26.70503 \quad 409892773$

$33.45278 \quad 49657 \quad 6721$

42.52444756601840

$\begin{array}{ll}14 & 12 \\ 14 & 13\end{array}$

15

$0.24503 \quad 301509310$

$0.82408 \quad 222004784$

1.74187240076449

3.00913245901792

4.64163406659064

6.66134149662717

9.09830270377053

$11.99370 \quad 343318574$

$15.40498 \quad 803105145$

19.41499282816615

$24.14975 \quad 795202928$

29.81810511967132

$36.81962 \quad 425699721$

$46.17743 \quad 001698345$

0

5

5

50

$\begin{array}{rr}0 & 50 \\ 0 & 5\end{array}$

0

$\begin{array}{lr}0 & 5 \\ 0 & 50 \\ 0 & 50 \\ 0 & 50 \\ 0 & 50 \\ 0 & 5 \\ 0 & 5 \\ 1 & 7 \\ 1 & 119 \\ 1 & 1890 \\ 1 & 2920 \\ 1 & 4460 \\ 1 & 6760 \\ 1 & 1060\end{array}$


TABLE II. Roots of $W_{n}(\epsilon / T)$-Continued

$\begin{array}{lr}n & l \\ 16 & 1 \\ 16 & 2 \\ 16 & 3 \\ 16 & 4 \\ 16 & 5 \\ 16 & 6 \\ 16 & 7 \\ 16 & 8 \\ 16 & 9 \\ 16 & 10 \\ 16 & 11 \\ 16 & 12 \\ 16 & 13 \\ 16 & 14 \\ 16 & 15\end{array}$

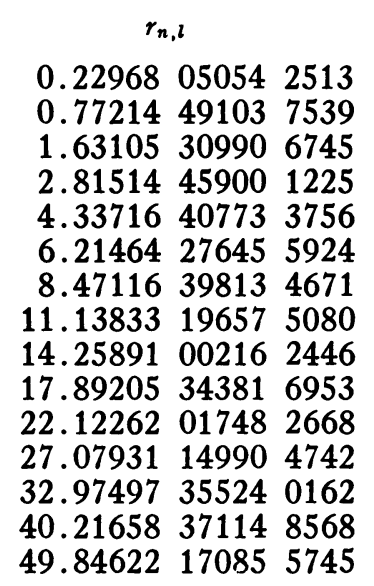

$\begin{array}{cr}C & e_{n, l} \\ 0 & 5 \\ 0 & 5 \\ 0 & 50 \\ 0 & 50 \\ 0 & 5 \\ 0 & 5 \\ 0 & 5 \\ 1 & 6 \\ 1 & 102 \\ 1 & 1600 \\ 1 & 2450 \\ 1 & 3680 \\ 1 & 5450 \\ 1 & 8060 \\ 1 & 1240\end{array}$

$\begin{array}{lr}17 & 1 \\ 17 & 2 \\ 17 & 3 \\ 17 & 4 \\ 17 & 5 \\ 17 & 6 \\ 17 & 7 \\ 17 & 8 \\ 17 & 9 \\ 17 & 10 \\ 17 & 11 \\ 17 & 12 \\ 17 & 13 \\ 17 & 14 \\ 17 & 15 \\ 17 & 16\end{array}$

0.21614030523945

0.72638824325183

1.53359316037353

2.64497099861195

4.07097816088018

5.82585551510563

7.92850418530666 $10.40380 \quad 828995104$ $13.28466 \quad 107070697$ 16.61517321686805 $20.45600 \quad 60200 \quad 3371$ 24.89384702533575 30.05986292016784 36.17069454363216 43.64036518415001 53.52915116025465

$\begin{array}{lr}0 & 5 \\ 0 & 50 \\ 0 & 50 \\ 0 & 50 \\ 0 & 50 \\ 0 & 50 \\ 0 & 5 \\ 1 & 5 \\ 1 & 88 \\ 1 & 1380 \\ 1 & 2090 \\ 1 & 30900 \\ 1 & 45000 \\ 1 & 65600 \\ 1 & 9540 \\ 1 & 14300\end{array}$

$\begin{array}{lr}18 & 1 \\ 18 & 2 \\ 18 & 3 \\ 18 & 4 \\ 18 & 5 \\ 18 & 6 \\ 18 & 7 \\ 18 & 8 \\ 18 & 9 \\ 18 & 10 \\ 18 & 11 \\ 18 & 12 \\ 18 & 13 \\ 18 & 14 \\ 18 & 15 \\ 18 & 16 \\ 18 & 17\end{array}$

$$
\begin{array}{rrr}
0.20410 & 91085 & 7933 \\
0.68576 & 75894 & 9453 \\
1.44719 & 86793 & 8049 \\
2.49443 & 08859 & 6248 \\
3.83615 & 60319 & 9932 \\
5.48411 & 54208 & 4047 \\
7.45372 & 29495 & 8128 \\
9.76498 & 43667 & 5180 \\
12.44386 & 10519 & 8395 \\
15.52434 & 30012 & 1457 \\
19.05171 & 87593 & 8568 \\
23.08800 & 07008 & 7174 \\
27.72155 & 63462 & 7824 \\
33.08585 & 98280 & 6407 \\
39.40115 & 37933 & 0861 \\
47.08820 & 96546 & 0141 \\
57.22481 & 18319 & 1492
\end{array}
$$

$\begin{array}{lrr}0 & & 5 \\ 0 & & 5 \\ 0 & & 50 \\ 0 & & 50 \\ 0 & & 50 \\ 0 & & 5 \\ 0 & & 5 \\ 0 & & 5 \\ 1 & & 77 \\ 1 & & 1200 \\ 1 & & 1810 \\ 1 & & 26700 \\ 1 & & 38500 \\ 1 & & 54800 \\ 1 & & 77500 \\ 1 & & 1 \\ 1 & & 11000 \\ 1 & & 16300\end{array}$


TABLE II. Roots of $W_{n}(\epsilon / T)$-Continued

$\begin{array}{ll}n & l \\ 19 & 1 \\ 19 & 2 \\ 19 & 3 \\ 19 & 4 \\ 19 & 5 \\ 19 & 6 \\ 19 & 7 \\ 19 & 8 \\ 19 & 9 \\ 19 & 10 \\ 19 & 11 \\ 19 & 12 \\ 19 & 13 \\ 19 & 14 \\ 19 & 15 \\ 19 & 16 \\ 19 & 17 \\ 19 & 18\end{array}$

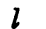

\begin{tabular}{ccc}
\multicolumn{3}{c}{$r_{n, l}$} \\
0.19334 & 77686 & 7901 \\
0.64946 & 18107 & 9655 \\
1.37007 & 54867 & 1706 \\
2.36027 & 76257 & 7508 \\
3.62737 & 58656 & 5560 \\
5.18117 & 49562 & 2015 \\
7.03443 & 06230 & 6566 \\
9.20350 & 66842 & 8214 \\
11.70932 & 47926 & 7879 \\
14.57876 & 11306 & 9283 \\
17.84675 & 98274 & 1882 \\
21.55965 & 87534 & 0514 \\
25.78070 & 10050 & 5240 \\
30.59981 & 44865 & 1918 \\
36.15265 & 02790 & 4671 \\
42.66288 & 77797 & 8564 \\
50.55778 & 33977 & 1778 \\
60.93200 & 77265 & 0073
\end{tabular}

C

0

0

0

0

0

0

0

0

0.18366517309616

0.61681638212673

1.30079940296677

2.23994939900886

$3.44047 \quad 19428 \quad 8150$

4.91064587967473

6.66116061205821

8.70559717712802

11.06110868357575

13.74939412008341

16.79812300595300

20.24308496330246 24.13156726760267 $28.52794 \quad 79713 \quad 1414$ 33.52361761728890 $39.2562942907 \quad 8263$ $\begin{array}{ll}45.95295 & 06486 \quad 3834\end{array}$ $54.04709 \quad 30247$
0194 64.64971243781605

$\begin{array}{rr}21 & 1 \\ 21 & 2 \\ 21 & 3 \\ 21 & 4 \\ 21 & 5 \\ 21 & 6 \\ 21 & 7 \\ 21 & 8 \\ 21 & 9 \\ 21 & 10 \\ 21 & 11 \\ 21 & 12 \\ 21 & 13 \\ 21 & 14\end{array}$
0.17490675238661
0.58730308063812
1.23822510183420
2.13139626007712
3.27213313351677
$4.6674944658 \quad 8837$
6.32653619767362
$8.26067 \quad 095201371$
10.48416738120829
13.01484877215828
$\begin{array}{llll}15.87508 & 70127 & 1499\end{array}$
19.09325190760624
$\begin{array}{llll}22.70589 & 38881 & 7321\end{array}$
26.76117022937952

C



$e_{n, l}$

15900

500

50

50

50

612

9450

14100

10

15

20

28

39

53

73

105

50

50

500

500

500

500

500

50

55

8470

126000

18

26 
TABLE II. Roots of $W_{n}(\epsilon / T)$-Continued

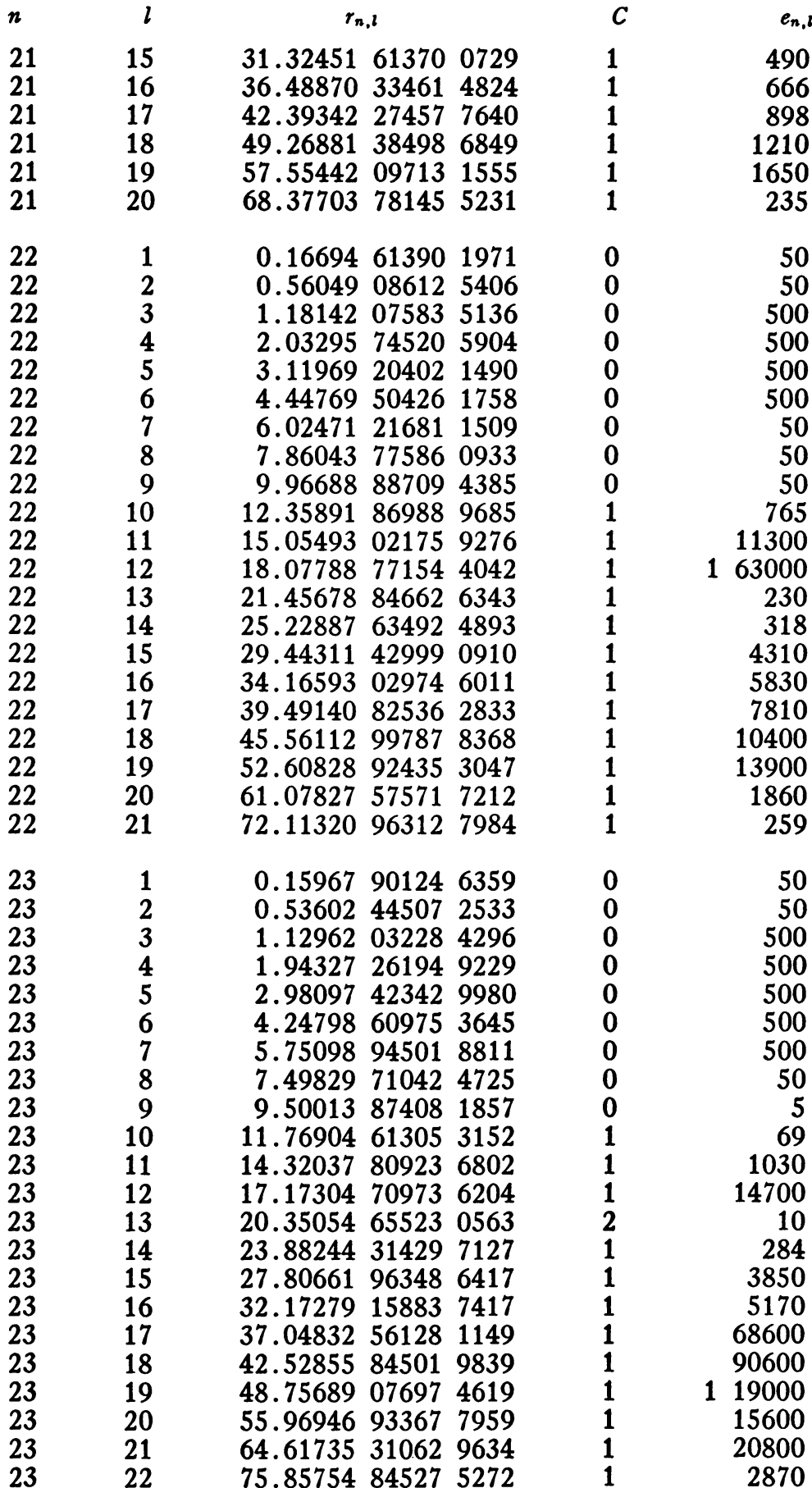


Table II. Roots of $W_{n}(\epsilon / T)$-Continued




TABLE II. Roots of $W_{n}(\epsilon / T)$-Continued

$\begin{array}{lr}n & l \\ 26 & 5 \\ 26 & 6 \\ 26 & 7 \\ 26 & 8 \\ 26 & 9 \\ 26 & 10 \\ 26 & 11 \\ 26 & 12 \\ 26 & 13 \\ 26 & 14 \\ 26 & 15 \\ 26 & 16 \\ 26 & 17 \\ 26 & 18 \\ 26 & 19 \\ 26 & 20 \\ 26 & 21 \\ 26 & 22 \\ 26 & 23 \\ 26 & 24 \\ 26 & 25\end{array}$

\begin{tabular}{rll}
\multicolumn{4}{c}{$r_{n, l}$} \\
2.63069 & 31145 & 8470 \\
3.74487 & 77262 & 0273 \\
5.06340 & 83123 & 3855 \\
6.59177 & 56068 & 7320 \\
8.33662 & 63598 & 0514 \\
10.30594 & 30256 & 1368 \\
12.50927 & 80113 & 1609 \\
14.95806 & 12826 & 7794 \\
17.66600 & 89930 & 4484 \\
20.64967 & 47456 & 6110 \\
23.92920 & 78044 & 9273 \\
27.52942 & 09021 & 3584 \\
31.48133 & 78942 & 1101 \\
35.82451 & 67628 & 4751 \\
40.61069 & 00156 & 5943 \\
45.90978 & 68582 & 2976 \\
51.82061 & 58754 & 0514 \\
58.49167 & 48142 & 7643 \\
66.16744 & 93598 & 1048 \\
75.31508 & 13581 & 0590 \\
87.13389 & 48199 & 8148
\end{tabular}



0.13600125704444

0.45636935813000

0.96118102730921

1.65214289298375

2.53167767949454

3.60294557808908

4.86990608262376

6.33740315572642

8.01127625438048

9.89850348307608

12.00738613818993

14.34778788105506

16.93144762042762

$19.7723941164 \quad 6035$

$22.88750 \quad 43962 \quad 7852$

$26.29727 \quad 100297123$

30.02688184387846

$34.10778 \quad 47142 \quad 1721$

38.58003519064629

$\begin{array}{llll}43.49597 \quad 58235 & 1797\end{array}$

$48.92632 \quad 265885339$

54.97096302530608

$\begin{array}{llll}61.77999 & 23077 \quad 3237\end{array}$

69.60052545734751

$\begin{array}{llll}78.90479 & 29547 \quad 6498\end{array}$

90.90552809951289

$27 \quad 26$

0.13114020484340

0.44002684102122

0.92665899797975

1.59256143539651

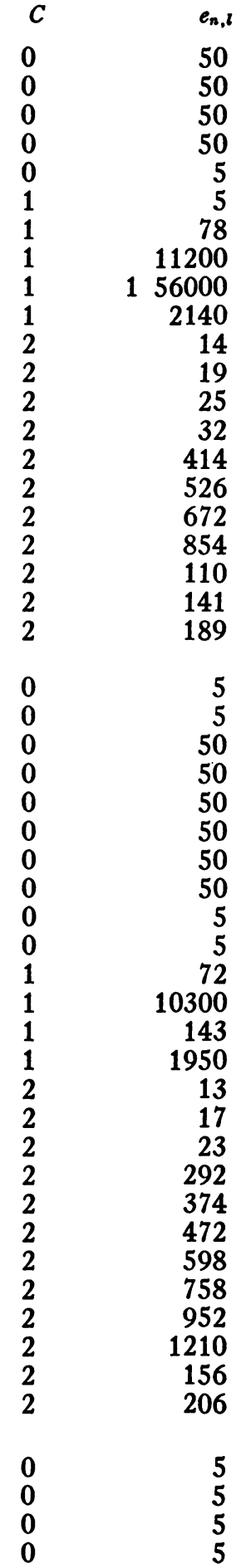


TABLE II. Roots of $W_{n}(\epsilon / T)$-Continued

\begin{tabular}{|c|c|c|c|c|c|}
\hline$n$ & $l$ & & $n, 6$ & $C$ & $e_{n, l}$ \\
\hline 28 & 5 & 2.43989 & 885988214 & 0 & 5 \\
\hline 28 & 6 & 3.47148 & 987678727 & 0 & 5 \\
\hline 28 & 7 & 4.69085 & 736862107 & 0 & 5 \\
\hline 28 & 8 & 6.10229 & 806635926 & 0 & 5 \\
\hline 28 & 9 & 7.71097 & 272091486 & 0 & 5 \\
\hline 28 & 10 & 9.52302 & 142148264 & 0 & 5 \\
\hline 28 & 11 & 11.54571 & 079832683 & 1 & 7 \\
\hline 28 & 12 & 13.78762 & 260830963 & 1 & 10 \\
\hline 28 & 13 & 16.25889 & 714832334 & 1 & 132 \\
\hline 28 & 14 & 18.97155 & 081919327 & 1 & 1800 \\
\hline 28 & 15 & 21.93989 & 617095973 & 2 & 12 \\
\hline 28 & 16 & 25.18110 & 697432692 & 2 & 16 \\
\hline 28 & 17 & 28.71599 & 402248103 & 2 & 21 \\
\hline 28 & 18 & 32.57009 & 650764767 & 2 & 266 \\
\hline 28 & 19 & 36.77526 & 283376414 & 2 & 338 \\
\hline 28 & 20 & 41.37202 & 267090563 & 2 & 430 \\
\hline 28 & 21 & 46.41330 & 392702954 & 2 & 5380 \\
\hline 28 & 22 & 51.97058 & 205383640 & 2 & 6760 \\
\hline 28 & 23 & 58.14479 & 024567904 & 2 & 8480 \\
\hline 28 & 24 & 65.08757 & 798116239 & 2 & 10600 \\
\hline 28 & 25 & 73.04862 & 351081621 & 2 & 1330 \\
\hline 28 & 26 & 82.50512 & 467565939 & 2 & 171 \\
\hline 28 & 27 & 94.68291 & 125829295 & 2 & 224 \\
\hline 29 & 1 & 0.12661 & 477726314 & 0 & 5 \\
\hline 29 & 2 & 0.42481 & 568942803 & 0 & 5 \\
\hline 29 & 3 & 0.89453 & 699672982 & 0 & 5 \\
\hline 29 & 4 & 1.53714 & $63597 \quad 6714$ & 0 & 5 \\
\hline 29 & 5 & 2.35458 & 600740362 & 0 & 5 \\
\hline 29 & 6 & 3.34938 & 089434731 & 0 & 5 \\
\hline 29 & 7 & 4.52468 & 000680930 & 0 & 5 \\
\hline 29 & 8 & 5.88431 & 365745907 & 0 & 5 \\
\hline 29 & 9 & 7.43286 & 738473363 & 0 & 5 \\
\hline 29 & 10 & 9.17577 & 578205631 & 0 & 5 \\
\hline 29 & 11 & 11.11944 & 122769518 & 1 & 6 \\
\hline 29 & 12 & 13.27138 & 444559570 & 1 & 9 \\
\hline 29 & 13 & 15.64043 & 655270260 & 1 & 123 \\
\hline 29 & 14 & 18.23698 & 621114301 & 1 & 1670 \\
\hline 29 & 15 & 21.07330 & 142714752 & 2 & 11 \\
\hline 29 & 16 & 24.16395 & 462798314 & 2 & 15 \\
\hline 29 & 17 & 27.52639 & 399715279 & 2 & 19 \\
\hline 29 & 18 & 31.18172 & 743314674 & 2 & 244 \\
\hline 29 & 19 & 35.15582 & 502559695 & 2 & 308 \\
\hline 29 & 20 & 39.48091 & 565241023 & 2 & 3900 \\
\hline 29 & 21 & 44.19798 & 254346970 & 2 & 4880 \\
\hline 29 & 22 & 49.36051 & 710530558 & 2 & 6100 \\
\hline 29 & 23 & 55.04072 & 955775849 & 2 & 7580 \\
\hline 29 & 24 & 61.34056 & 902216674 & 2 & 9440 \\
\hline 29 & 25 & 68.41319 & 891673268 & 2 & 11700 \\
\hline 29 & 26 & 76.51079 & $93357 \quad 6897$ & 2 & 14700 \\
\hline 29 & 27 & 86.11542 & 169924401 & 2 & 1860 \\
\hline 20 & 28 & 98.46569 & 766293931 & 2 & 242 \\
\hline
\end{tabular}




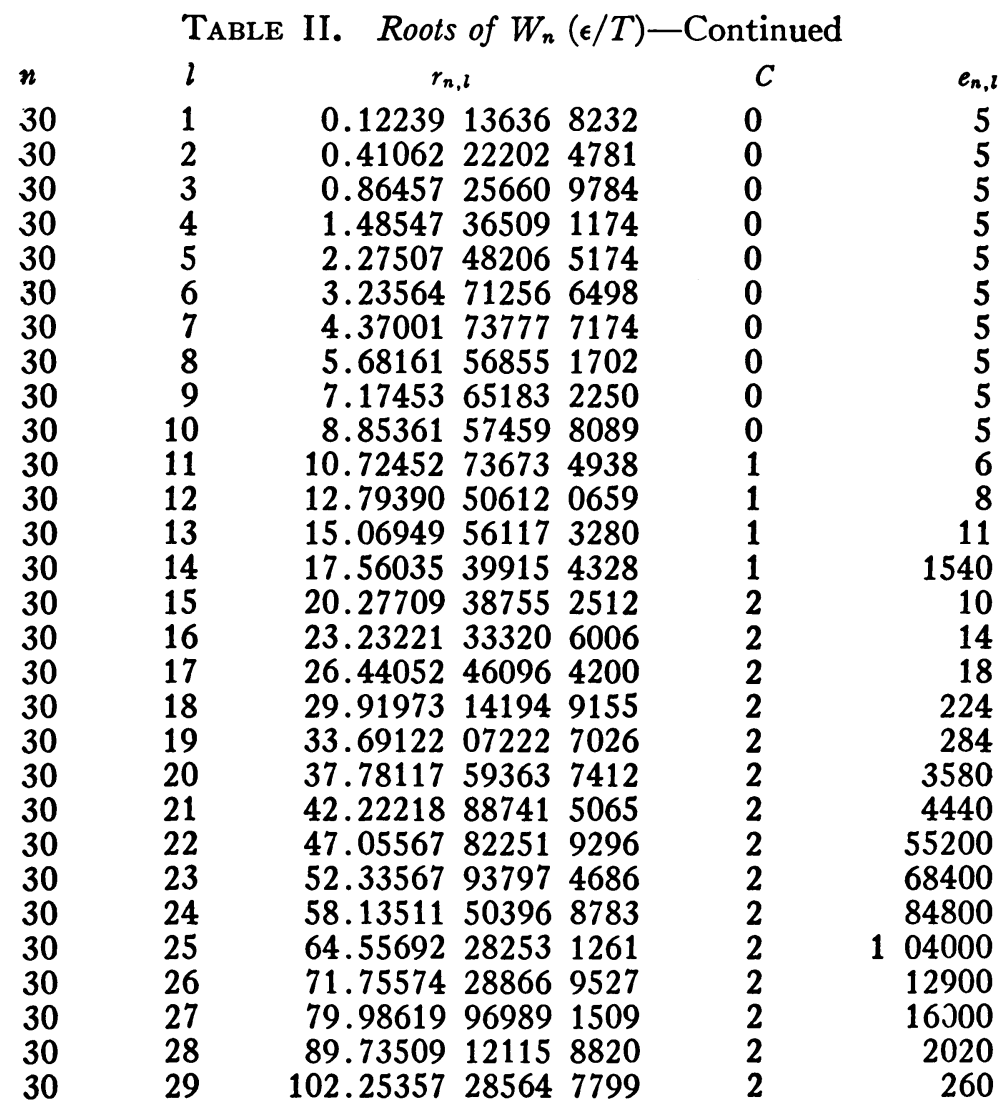

Legend:

$r_{n, l}:$ the $l$ th root of the $n$-th order polynomial, $W_{n}$. The estimated error bound is

$$
\mid r_{n, l}-\text { root } \mid \leqq e_{n, l} \times 10^{-14}
$$

The significance of the numbers in column $C$ is:

$$
\begin{aligned}
& \text { 0: the root was computed explicitly } \\
& \text { 1: the root was computed as } r_{n, l}=10 / r_{n, l} \\
& \text { 2: the root was computed as } r_{n, l}=20 / \bar{r}_{n, l}
\end{aligned}
$$

The formulae of equation (4) can be verified by induction based on the actual formal construction of successive derivatives of $\exp (-\epsilon / T)[1]$. After these relations have been established they can be used with a simple induction argument to establish the correctness of explicit formulae which are convenient for other purposes, albeit not for calculations

$$
b_{n, l}=\frac{(-1)^{n-l} l(l+1)^{2} \cdots(n-1)^{2} n}{(n-l) !} .
$$

The roots were located by the method of false position [2], which depends upon evaluation of the polynomials for successive approximations to the roots. The evaluations were computed by synthetic division using double precision arithmetic on the ORDVAC computer at the Aberdeen Proving Ground. The polynomials were calculated in one of three forms as described in the legend to Table II. This was necessary since the original form of the polynomial was relatively insensitive to changes in approximations for certain roots; that is, the remainder was too small 


\section{TABLE III. Symmetric Function Check on Roots}

\begin{tabular}{|c|c|c|}
\hline$n$ & $b_{n, n-1}$ & $d_{n}$ \\
\hline 2 & 2 & 0 \\
\hline 3 & 6 & 0 \\
\hline 4 & 12 & - \\
\hline 5 & 20 & - \\
\hline 6 & 30 & - \\
\hline 7 & 42 & + \\
\hline 8 & 56 & - \\
\hline 9 & 72 & + \\
\hline 10 & 90 & - \\
\hline 11 & 110 & + \\
\hline 12 & 132 & + \\
\hline 13 & 156 & + \\
\hline 14 & 182 & + \\
\hline 15 & 210 & $-\quad 371$ \\
\hline 16 & 240 & -1263 \\
\hline 17 & 272 & -13059 \\
\hline 18 & 306 & +21289 \\
\hline 19 & 342 & $\begin{array}{l}+927 \\
+\quad 9\end{array}$ \\
\hline 20 & 380 & + \\
\hline 21 & 420 & -6267 \\
\hline 22 & 462 & +39096 \\
\hline 23 & 506 & -2411 \\
\hline 24 & 552 & -55267 \\
\hline 25 & 600 & -5915 \\
\hline 26 & 650 & +23010 \\
\hline 27 & 702 & $\begin{array}{r}1762 \\
\end{array}$ \\
\hline 28 & 756 & $\begin{array}{r}223 \\
\end{array}$ \\
\hline 29 & 812 & -4329 \\
\hline Q & 870 & -18880 \\
\hline
\end{tabular}

Legend: $b_{n, n-1}-\sum_{l=1}^{n} r_{n, l}=d_{n} \times 10^{-14}$

compared with the largest term in the synthetic division schema. When this occurred, one of the other forms of the polynomial was used in order to define the root more accurately.

The error estimates of Table II were based upon locating the root between two approximate values which gave remainders of opposite sign. Although this method of locating roots is inherently self-checking, a further numerical test was made by computing the symmetric function summations of Table III.

New York University,

Edwin S. Campbell

New York

Ballistic Research Laboratories,

E. M. FischbaCh

Aberdeen, Maryland

University of Wisconsin

J. O. HIRSCHFELDER

Milwaukee, Wisconsin

1. J. O. Hirschfelder \& Edwin S. Campbell, Analytical (power series) Solutions of Flame Propagation (CM-784), University of Wisconsin Naval Research Laboratory, May 27, $1953, p$. $17 \mathrm{ff}$.

2. F. B. Hildebrand, Introduction to Numerical Analysis, McGraw-Hill Book Co., New York, 1956, p. 446. 